\title{
Reversible heart failure: toxins, tachycardiomyopathy and mitochondrial abnormalities
}

\author{
Paul D Morris, ${ }^{1}$ Tim Robinson, ${ }^{2}$ Kevin S Channer ${ }^{3}$
}

${ }^{1}$ Department of Cardiology, Sheffield Teaching Hospitals NHS Trust, Sheffield, UK ${ }^{2}$ Department of Cardiology, Nottingham University Hospitals NHS Trust, Nottingham, UK ${ }^{3}$ Department of Cardiology, Royal Hallamshire Hospital, Sheffield, UK

\section{Correspondence to} Dr Paul Morris, Cardiology, Sheffield Teaching Hospitals NHS Trust, Herries Road, Sheffield S5 7AU, UK; paulmorris@doctors.org.uk

Received 16 December 2011 Accepted 27 May 2012 Published Online First 28 June 2012

\begin{abstract}
Heart failure is usually a relentless condition associated with a poor prognosis. Triggered by a physiological insult, maladaptive neurohumoral processes result in an ever-spiralling deterioration of cardiovascular function. However, there are certain underlying conditions which are associated with a temporary reduction in contractile function leading to reversible heart failure. These conditions affect a relatively small number of patients when compared with heart failure secondary to inherited cardiomyopathies and ischaemic heart disease. There are two broad mechanisms responsible for reversible myocyte dysfunction: acute inflammatory activation in which cytokines depress myocyte function, and toxic effects in which there is impairment of intra-cellular energetics. In this review, we discuss reversible heart failure caused by toxic effects. These effects can be caused by drugs (prescribed and illicit) and by tachycardic arrhythmia (tachycardiomyopathy), and are caused by abnormalities of mitochondrial function and myocytic calcium processing. The underlying pathological mechanisms, clinical features and management options are discussed, illustrated by clinical case studies.
\end{abstract}

\section{INTRODUCTION}

Heart failure is usually a relentlessly progressive condition characterised by dyspnoea, oedema and cachexia. The prognosis for patients with heart failure remains poor with a 5 -year survival rate from first presentation, of only $45-59 \% .{ }^{1}$ This is despite advances in pharmacological therapy with $\beta$-blockers, ACE inhibitors, spironolactone and device-based, resynchronisation therapy. However, there are some more uncommon causes of heart failure in which the clinical course is variable, and from which patients may recover in terms of both clinical status and left ventricular function. This is in contrast with heart failure caused by inherited cardiomyopathy, or ischaemic heart disease, which rarely demonstrate significant reversibility, and which are more common.

We have previously discussed the role of inflammation in the aetiology of reversible heart failure. ${ }^{2}$ Transient heart failure may be seen in conditions associated with acute pro-inflammatory cytokine activation, such as severe infections, auto-immune inflammatory conditions (eg, rheumatoid arthritis), in some women during the peripartum period and in multisystem disease states requiring intensive care management. Box 1 outlines a summary of this article. In this review, we discuss another mechanism for reversible heart failure seen in patients with incessant tachycardia and after ingestion of myocardial toxins, which may include prescribed and illicit drugs. The underlying mechanisms responsible for the transient myocardial suppression observed in these circumstances are centred around abnormalities of mitochondrial function. Mitochondrial DNA is more susceptible than nuclear DNA to stress-induced oxidative damage. Toxins and incessant tachycardic states negatively influence the enzymes involved in the tricarboxylic acid cycle, disturb the balance of oxidative and antioxidant enzymes, and impair the uptake and binding of calcium within the mitochondria. These mitochondrial abnormalities impair the synthesis of adenosine-5'-triphosphate (ATP) in the myocyte, and result in impaired contractility. A greater understanding of these underlying mechanisms may lead to novel drug therapies for heart failure. In this review, we use clinical case examples to consider the effects of mitochondrial toxins and incessant tachycardia on mitochondrial metabolism, in the context of reversible cardiomyopathy.

\section{ALCOHOL}

Chronic alcohol abuse is a well recognised cause of impaired cardiac contractility which may reverse with abstinence. It is associated with significant biventricular systolic dysfunction and dilatation. Left ventricular hypertrophy may also be observed, but is likely to be mediated by hypertension which accompanies chronic alcohol abuse. A combination of increased left ventricular end diastolic diameter (left ventricular dilatation) and hypertrophy accounts for the increase in left ventricular mass, observed even in asymptomatic individuals. ${ }^{3}{ }^{4}$ The main problem, however, is loss of myocardial contractility. Alcohol damages mitochondria and is a myocardial suppressant. As cardiac myocytes do not contain the enzyme alcohol dehydrogenase, the toxic effects of alcohol may result directly from the toxicity of ethanol. Peripherally synthesised acetaldehyde is also toxic to the myocyte. Ethanol inhibits mitochondrial respiration by impairing the function of enzymes in the tricarboxylic acid cycle. It also impairs the uptake and binding of calcium in the mitochondria. Acetaldehyde reduces myocardial protein synthesis and inhibits calcium-activated myofibrillar ATPase. The combination of these effects results in diminution in contractility and function. ${ }^{5}$

\section{ILLUSTRATIVE CASE}

A 39-year-old man was admitted with a history of dry cough, worsening breathlessness, weight gain and abdominal swelling. He was a smoker of 20 cigarettes daily and admitted to drinking over 70 units of alcohol per week. Examination revealed signs of severe congestive cardiac failure with 
Box 1 Reversible heart failure: the role of inflammatory activation: summary ${ }^{2}$

- Acute inflammatory activation can cause transient suppression of myocardial cell function.

- The underlying mechanism appears to be mediated via the activation of inflammatory cytokines and autoantibodies.

- These effects can be abrogated, at least partially, by immune modulating therapies such as intravenous immunoglobulin therapy.

- Supportive treatment while the underlying disease process is corrected results in cardiac recovery.

- Inflammatory activation as part of maladaptive physiology in patients with chronic heart failure is associated with a worse prognosis.

resting tachycardia of $126 \mathrm{bpm}$, blood pressure (BP) 131/90 and raised central venous pressure to $10 \mathrm{~cm}$. There was a summation gallop and bilateral pitting oedema. Chest radiograph demonstrated cardiomegaly and pulmonary congestion with fluid in the horizontal fissure. The mean cell volume and gamma-GT were raised, and there was ECG and echocardiographic evidence of left ventricular hypertrophy and severe global left and right ventricular systolic dysfunction with a left ventricular end diastolic dimension (LVEDD) of $57 \mathrm{~mm}$. He was treated with ACE inhibitors and low-dose $\beta$ blockade. After 3 months of abstinence, he reported normal exercise capacity with no overt clinical signs of cardiac dysfunction. At 12 months, echocardiography revealed normal left and right ventricular dimensions (LVEDD $47 \mathrm{~mm}$ ) and ejection fraction. He has remained abstinent and well since, and an echocardiogram over 18 months later showed a further reduction in LVEDD to $40 \mathrm{~mm}$.

\section{COMMENT}

Although treated with ACE inhibitors and $\beta$ blockade, the key intervention here was abstinence from alcohol. Current evidence suggests that a mild to moderate intake of alcohol is beneficial to the heart. The Spanish cohort of the European Prospective Investigation into Cancer and Nutrition (EPIC) trial studied over 50000 men and women over a 10-year period and found that in men, the frequency of coronary events was inversely proportional to the amount of alcohol consumed, with coronary events being more than $30 \%$ less likely in drinkers than non-drinkers. However, there was no additional benefit in men drinking 'high' and 'very high' amounts of alcohol. ${ }^{6}$ There was no significant relationship in women which might be explained by a lower event rate in the female cohort. At higher levels of consumption, the cardiac response to alcohol is highly variable. Some hearts tolerate higher levels of consumption than others. Although environmental factors, such as the effects of prescribed and illicit drugs have an additive effect, there is evidence of a genetic predisposition to alcohol-induced cardiomyopathy. One study of alcoholic men demonstrated a specific polymorphism of the ACE genotype in $57 \%$ of alcoholic persons with a left ventricular ejection fraction (LVEF) of $<50 \%$ but only in $7 \%$ of alcoholics with normal cardiac function. ${ }^{7}$ Even with complete abstinence, the degree of reversibility of cardiac function is variable, although, ensuring patients are truly abstinent is not always easy.

\section{Sympathomimetic drugs (cocaine and amphetamines)}

Recreational sympathomimetic drugs include cocaine, amphetamines and ecstasy. They have broadly similar stimulant effects, inducing euphoria, and a heightened psychological and physical state. Illegal use of sympathomimetic agents is highly prevalent in the UK with use steadily rising year on year. After cannabis, sympathomimetic compounds are the most confiscated class of drugs in the UK. An estimated 16.5\% of people between 15 and 34 years of age admit to some level of use. ${ }^{8}$

Cocaine is a crystalline tropane alkaloid that is obtained from the leaves of the coca plant. Its anaesthetic, stimulatory, medicinal and aphrodisiac properties have been recognised for centuries. However, cocaine and its free base 'crack-cocaine' are highly addictive, and are potent sympathomimetic agents with potentially lethal cardiovascular effects. ${ }^{9}$ Cocaine may cause an acute or chronic fall in left ventricular performance. Acute left ventricular systolic and diastolic dysfunction is caused, at least in part, by its sodium and potassium channel-blocking effects which impair myocyte calcium transport. ${ }^{10}$ Focal myocarditis, myocyte necrosis and myofibrillar loss have been demonstrated in myocardial biopsy specimens in the context of acute cocaine toxicity ${ }^{11}$ Cocaine blocks presynaptic uptake of catecholamines and dopamine, leading to postsynaptic over-activation of sympathetic and dopaminergic receptors. ${ }^{12}$ It also has a small central effect, causing noradrenaline (NA) release from the adrenal medulla. ${ }^{13}$ In actively intoxicated users, the level of circulating NA can be as much as five times higher than normal. ${ }^{10}$ Cocaine causes alterations in endothelial cytokine production and in circulating leucocytes. These changes activate the transcription of genes responsible for changing the composition of myocardial collagen and myosin, and inducing myocyte apoptosis. Chronic left ventricular systolic dysfunction is seen in $7 \%$ of asymptomatic long-term cocaine users. ${ }^{11}$ Cocaine has a short serum half-life of $30-80 \mathrm{~min}$ but can be detected in the urine for up to 2 weeks after administration, providing ample opportunity for clinicians to detect its use. ${ }^{14}$

Amphetamines are synthetic compounds named from their full chemical name, $\boldsymbol{\alpha}$-methyl-phenethylamine. They represent a range of psychoactive drugs, prodrugs and derivatives, including metamphetamine and ecstasy. In contrast with cocaine, amphetamines freely cross the blood-brain barrier and act mainly via the central nervous system to release NA, dopamine and serotonin. Amphetamines are used clinically in managing conditions such as attention-deficit hyperactivity disorder, postural orthostatic tachycardia syndrome and in some patients with traumatic brain injury. Recreationally, this class of drug is ingested, applied to the nasal or gingival mucous membranes, smoked or injected intravenously. Amphetamines are taken illicitly to achieve a 'high' which reduces somnolence, allowing users to stay awake for long periods. Other desirable effects include enhanced sporting or work performance and improved self-confidence. Physical signs include hyperactivity, flushing, hypertension, muscular twitching and insomnia. Psychological effects include anxiety, increased libido, heightened alertness, aggression, paranoia, grandiosity and even frank psychosis. These effects can be augmented by the concomitant use of alcohol or cannabis which alter the metabolism of amphetamines. Unlike cocaine, amphetamines lack the ability to block sodium and potassium channels. Amphetamines may induce myocardial ischaemia by a combination of factors. Focal, or more rarely, diffuse coronary spasm may occur. Sympathetic stimulation results in increased myocardial oxygen demand, and both, cocaine and amphetamines can induce a procoagulant 
effect. ${ }^{10} 15$ Some amphetamine users ingest bicarbonate in order to prolong drug excretion and lengthen the 'high', an effect mediated via alkalinisation of the blood.

\section{ILLUSTRATIVE CASE}

A 35-year-old lady with no prior cardiac history was admitted to the accident and emergency department, hypotensive, tachypnoeic and coughing up copious amounts of frothy pink sputum. Her chest radiograph confirmed severe pulmonary oedema, and an echocardiogram on the intensive care unit demonstrated a severely globally impaired left ventricle. Two hours prior to her admission, her partner had 'treated her', consensually, to an intravenous injection of approximately $1.5 \mathrm{~g}$ of amphetamine. She had a history of intravenous drug use and was chronically treated with mirtazepine and methadone. She required intubation and ventilation on the intensive care unit and inotropic support with milrinone and BP support with the vasopressor, norepinephrine. She was actively cooled due to persistent fever. Her condition continued to decline and within $24 \mathrm{~h}$ a decision was made to provide additional cardiovascular support with an intra-aortic balloon pump. Her ECG demonstrated subtle ST elevation in the inferior and lateral leads, and a depressed PR segment in aVR, thought to reflect a degree of pericarditis. Thirtysix hours later, the intra-aortic balloon pump was removed but she continued to require inotropic and vasopressor support. Four days into her admission she was commenced on ACE inhibitors and $\beta$ blockers. After 5 days, she no longer required inotropic support and a transoesophageal echocardiogram demonstrated a dilated and severely impaired left ventricle with severe mitral regurgitation and an LVEF of $<20 \%$. After 1 week, she had improved sufficiently to be transferred from intensive care. She continued to improve with slowly up-titrating doses of bisoprolol and ramipril. Three weeks after her admission, repeat echocardiography revealed a normal LVEDD of $43 \mathrm{~mm}$ and normal left ventricular function with no mitral regurgitation. She was discharged home shortly afterwards with no symptoms of cardiac failure. Twelve weeks later, she remained asymptomatic with normal echocardiography. She denied further use of illicit drugs.

\section{COMMENT}

This case demonstrates the acute cardiac toxicity of high-dose amphetamine use from a presumed normal to severely impaired left ventricle with severe pulmonary oedema in only a couple of hours. Severe spasm affecting all three epicardial arteries in their proximal segments is unlikely. Over the next $96 \mathrm{~h}$ the left ventricle had dilated resulting in severe functional mitral regurgitation. In $<3$ weeks, her clinical state and echocardiographic findings had completely normalised.

\section{TACHYCARDIA-MEDIATED CARDIOMYOPATHY (TMC)}

Arrhythmia is frequently the consequence of cardiomyopathy, but is often overlooked as the potential cause. Atrial flutter, atrial tachycardia, atrioventricular nodal re-entrant tachycardia, atrioventricular reciprocating tachycardia and ventricular tachycardia have all been shown to cause tachycardia-mediated cardiomyopathy. ${ }^{16}$ TMC is uncommon. In a series of 673 patients with dilated cardiomyopathy, only one case was attributed to incessant tachycardia. ${ }^{17}$ Animal models demonstrate that cardiac pacing at rates of $180-200 \mathrm{bpm}$ for 3 weeks will induce heart failure. There are several mechanisms that can cause myocardial dysfunction in TMC. Chronically high heart rates cause oxidative mitochondrial damage contributing to worsening heart failure. Mitochondrial DNA is more vulnerable to oxidative damage than nuclear DNA. A relative imbalance between pro-oxidant and antioxidant pathways may explain this observation. Inflammation also plays a role in the pathogenesis of tachycardia-induced cardiomyopathy. ${ }^{2}$ Experimental cardiac pacing in dogs produces an increase in myocardial monocyte infiltration, monocyte chemoattractant protein-1 expression, and renin-angiotensin system and local matrix metalloproteinase activity. The progressive loss of valuable cardiomyocytes in heart failure is attributed to apoptosis, a result of various ongoing metabolic and neurohumoral insults. The observed increase in apoptosis correlates with elevated norepinephrine levels. ${ }^{18}$ The rise in angiotensin II levels, tissue apoptosis, white blood cell infiltration and cell death are greater in atrial TCM than ventricular TCM. The observed ventricular fibrotic changes are thought to be related to elevated levels of transforming growth factor- $B .{ }^{19}$ Early recognition and prompt treatment appears to be the common theme in the management of patients with tachycardia-induced atrial and ventricular changes.

\section{ILLUSTRATIVE CASE}

A 44-yr-old man with a long history of palpitations presented with a 1-month history of progressive dyspnoea. On presentation to hospital, he was in clinical NYHA class IV heart failure, was tachycardic at $260 \mathrm{bpm}$, and had BP 106/72 mm Hg, JVP raised to $8 \mathrm{~cm}$ and pulmonary rales. CXR confirmed pulmonary oedema. ECG demonstrated a regular broad complex tachycardia. Adenosine was given which revealed underlying flutter waves and manifest pre-excitation consistent with a left free wall accessory pathway. In view of the cardiac failure, he was electrically cardioverted which restored sinus rhythm. Subsequent echocardiography demonstrated a dilated and poorly functioning left ventricle. Initial treatment with loop diuretics was followed by the commencement of ACE inhibition and $\beta$ blockade. Later cardiac MRI confirmed the echocardiographic findings with LVEF 46\% and LV end diastolic volume $246 \mathrm{ml} \mathrm{An}$ electrophysiological study confirmed the presence of a left postero-lateral accessory pathway and right atrial cavotricuspid isthmus-dependent flutter. Ablation of both the cavotricuspid isthmus and the accessory pathway was performed. Over the subsequent weeks, his heart failure symptoms resolved completely and a repeat cardiac MRI 3 months later demonstrated that left ventricular size and function had returned to normal (LVEF 65\%, LVEDV $200 \mathrm{ml}$ ) with no evidence of myocardial scarring.

\section{COMMENT}

When patients present with tachyarrhythmia and heart failure with little prior cardiac history, it may be impossible to know whether the cardiomyopathy is the cause or the consequence of the tachyarrhythmia. Clinical therapy should focus on both the heart failure and controlling the tachyarrhythmia (rate and/or rhythm if possible). An electrophysiological study should be considered, since ablation (if appropriate) offers a high cure rate. The presence of heart failure may restrict the pharmacological options in managing the arrhythmia. Only with the passage of time and optimum medical therapy of both, the heart failure and the arrhythmia, will it become clear whether the impairment of left ventricular function will recover and to what extent. The combination of arrhythmia and impaired contractile function should prompt the physician to consider anticoagulation to avoid thrombo-embolic complications.

\section{CYTOTOXIC DRUGS}

Cardiotoxicity occurs during treatment with many cytotoxic drugs. This may be the dose-limiting factor in cancer treatment 
and may, therefore, affect tumour response. Cardiotoxicity can also be responsible for long-term effects and may cause severe morbidity in surviving cancer patients. ${ }^{20}$ Different mechanisms of chemotherapy-induced cardiotoxicity have been proposed, ranging from cellular damage from oxygen free radicals, induced immunogenic reactions with antigen-presenting cells in the myocardium, to the effects of chemotherapeutic agents on cardiolipin which affects ATP synthesis in mitochondria. ${ }^{21} 22$

\section{ANTHRACYCLINES}

The association between anthracycline treatment and increased risk of heart failure has been known since the 1970s. The risk of heart failure depends on the cumulative dose received. ${ }^{23}$ The anti-cancer effects of anthracyclines are primarily mediated through inhibition of DNA synthesis, transcription and replication. Anthracyclines generate oxygen-derived free radicals using iron as a co-factor, and this impairs the mitochondrial respiratory chain. Free radicals damage proteins, lipids and DNA directly. Evidence suggests that increased myocyte apoptosis is related to the consequent increase in oxidative stress. ${ }^{24-27}$ This hypothesis is mainly based on in vitro experiments with only scant in vivo studies. The specific susceptibility of cardiac cells to oxidative stress may be due to relatively low levels of antioxidant enzymes in the heart. ${ }^{28}$ Oxidative stress results in the formation of oxidative free radicals which induce apoptosis. Another mechanism involves the influence of anthracyclines on calcium homeostasis. Oxidative stress can induce alterations in mitochondrial permeability which influences mitochondrial calcium transport. Changes in calcium transport can lead to impaired contractility, tissue injury and cell death. In vitro experiments have shown that doxorubicin treatment causes an irreversible decrease in mitochondrial calcium-loading capacity. ${ }^{29}$

\section{ILLUSTRATIVE CASE}

A 15-year-old boy was admitted with evidence of decompensated heart failure. At the age of four years, he had been treated for a rhabdomyosarcoma with a combination of carboplatin, ifosfamide, etoposide, vincristine, actinomycin and epirubicin (anthracycline). The cumulative dose of epirubicin was $600 \mathrm{mgs} / \mathrm{m}^{2}$. Echocardiography revealed a dilated left ventricle with severely reduced contractile function and an ejection fraction of $<20 \%$. He was referred for a cardiac transplant assessment, but improved on medical therapy in the interim period (furosemide, ACE inhibition, nitrate and digoxin). Twelve months later, he was reviewed in clinic, still on medical therapy. At this point, he was asymptomatic, and a repeat echocardiogram revealed normal pulmonary artery pressure, ventricular dimensions and left and right ventricular function. A $\beta$ blocker was then introduced and digoxin and nitrate were withdrawn. Five years later, he remained asymptomatic and was in full-time employment. The most recent echocardiogram (6 years after the initial presentation) demonstrated impaired diastolic function with only mild impairment of left ventricular function. Increased ventricular septal stiffness with diastolic dysfunction is characteristic of anthracycline-induced cardiomyopathy.

\section{COMMENT}

Acute, or subacute cardiotoxicity with anthracyclines is rare, and occurs during, or immediately following, infusion. It is usually transient and will attenuate after discontinuation of therapy. ${ }^{30}$ However, chronic effects can persist after discontin- uation of therapy, with all the features of cardiomyopathy. One of the commonest presentations of anthracycline-induced cardiomyopathy is in women treated for metastatic breast cancer. Direct toxic damage to the myocardium usually affects the inter-ventricular septum first. A range of damage is seen in patients exposed to this drug, but the majority do not develop clinically significant failure. There are a number of risk factors which increase the likelihood of developing heart failure after anthracycline therapy. In a retrospective study of 1097 patients with metastatic breast cancer treated with epirubicin, Ryberg et al found that $11.4 \%$ developed congestive cardiac failure with a $40 \%$ increase in risk for every $100 \mathrm{mg} / \mathrm{m}^{2}$ administered. ${ }^{31}$ Other factors which increased the risk of heart failure during treatment were: increasing age (28.5\% for every 10 years in age), a predisposition to cardiac disease, a prior (not adjuvant) history of mediastinal irradiation (208\%) or prior use of anti-hormonal treatments for breast cancer (87\%). By the age of 70 years, the risk of developing heart failure with the lowest dose of epirubicin given was $5 \%$, but this increased to nearly $20 \%$ for those given higher doses. The same authors also studied the time course for the development of heart failure after epirubicin therapy, ${ }^{32}$ showing that the median time to heart failure onset was 57 days (range 0-853 days). Previous irradiation directed over the left hemithorax increased the risk of death due to cardiac failure and reduced the median survival time. A French study of low-dose epirubicin reported lower frequencies of heart failure, but risk increased in women over 65 years of age, and in those with a body mass index $>27 \mathrm{~kg} / \mathrm{m}^{2}$. 33

Aside from cumulative dose reduction, attempts have been made to develop chemoprotectants to prevent anthracyclineinduced cardiotoxicity. Antioxidants used as free radical scavengers have not shown any cardioprotective effect. ${ }^{34} 35$ Iron chelators have been developed to reduce cardiotoxicity by binding intracellular iron and removing the iron from the anthracycline-iron complex, thus preventing free radical formation. Dexrazoxane was found to be the most promising agent reducing cardiac side effects from $31 \%$ to $15 \%(p<0.001)$ in one study, and from $24 \%$ to $4 \%$ in another $(p=0.02) .{ }^{36} 37$ However, there is a concern that the antioxidant effects of Dexrazoxane may reduce the anti-tumour efficacy of anthracyclines. Attempts have also been made to change the formulation of the anthracyclines, for example, to liposomal doxorubicin which may reduce toxicity. ${ }^{38}$

\section{TRASTUZUMAB (HERCEPTIN)}

Trastuzumab is a humanised anti-HER2 monoclonal antibody used in the treatment of breast cancer in patients with overexpression of human epidermal growth factor receptor type 2 (HER2). ${ }^{39} 40$ Trastuzumab alone is associated with cardiac toxicity and increases the risk of congestive heart failure when used in patients with early or with metastatic disease. When used in combination with or after an anthracycline drug, the risk of CHF is even higher. Studies of mutant mouse models have shown a pivotal role of the erbB2 gene in the postnatal heart. ${ }^{41}$ ErbB2-mediated pathways are lost in trastuzumab cardiotoxicity. These pathways normally blunt the effects of stress-signalling pathways activated by anthracyclines. ErbB2 protein-deficient mice are, therefore, particularly susceptible to the effects of haemodynamic overload and anthracyclines by developing left ventricular dysfunction. ErbB2 is also fundamental in triggering myocyte-survival pathways that are required during the activation of acute stress signals. After trastuzumab treatment, the loss of these survival cues can result in irreversible loss of cardiac myocytes resulting 
in overt cardiac dysfunction. ${ }^{42}$ However, the precise mechanism of the trastuzumab and anthracycline interaction remains unknown.

\section{ILLUSTRATIVE CASE}

A 50-year-old woman with metastatic breast carcinoma was referred with echocardiographic evidence of deteriorating left ventricular function. She had initially been treated with eight courses of palliative chemotherapy, including an anthracycline. At presentation, she was taking capecitabine and herceptin. Pretreatment LVEF was $>60 \%$ but had fallen to only $30-35 \%$ with an increase in LVEDD to $56 \mathrm{~mm}$ and associated functional moderate mitral regurgitation. It was felt that herceptin treatment may have unmasked occult damage induced by prior anthracycline therapy. Herceptin therapy was withheld and she was commenced on ACE inhibition and low-dose $\beta$-blocker therapy. After 4 weeks, there was echocardiographic improvement with an increase in ejection fraction to $\sim 40 \%$, and less mitral incompetence. Within 2 months, another echocardiogram demonstrated continued improvement with a LVEF of $50 \%$ and only mild mitral regurgitation. At this point, spironolactone was added. She was recommenced on herceptin after a 5 -month break with frequent echocardiographic monitoring. Twelve months after her original clinical cardiac deterioration, echocardiogram demonstrated a non-dilated left ventricle, trivial mitral regurgitation and an ejection fraction of $60 \%$ with no evidence of diastolic dysfunction.

\section{COMMENT}

Although this case demonstrates reversal of the presenting cardiomyopathy, she remains at high risk of recurrent cardiac damage from herceptin and will require ongoing monitoring of left ventricular function. Current guidelines suggest that patients receiving herceptin should have LVEF monitored by echocardiography, or by the radionucleotide MUGA scan every 12 weeks. The frequency of surveillance needs to be increased if there is evidence of deterioration. The risk of cardiotoxicity following trastuzumab administration is associated with older age, lower LVEF at onset of treatment, concomitant use of antihypertensive medications and in patients who had also received anthracyclines. ${ }^{43}$ The cardiac toxicity is much more common when trastuzumab and anthracyclines are given together or within a recent time span, therefore, concomitant administration of these two drugs should be avoided as recommended by recently proposed guidelines. ${ }^{44}$ Identification of patients at high risk, especially in the setting of trastuzumab in combination with an anthracycline, and optimisation of their cardiac risk factors is required to reduce the risk of cardiac toxicity with these drugs.

\section{MITOCHONDRIAL ABNORMALITIES IN HEART FAILURE}

The illustrative cases in this review demonstrate the role of toxins in the aetiology of cardiac failure. Most notably, it is the toxic effect upon the mitochondria within the cardiac myocyte that causes a reduction in cellular energetics resulting in decreased contractile function. There is then a tendency for the development of further abnormalities in mitochondrial metabolism especially relating to oxygen consumption and oxidative phosphorylation. Myocyte, and consequent cardiac work, requires a high rate of ATP hydrolysis, matched by ATP production through mitochondrial oxidative phosphorylation. Lower concentrations of ATP are found in patients with chronic heart failure. ${ }^{45}$ In addition to the drop in ATP content, the failing heart has abnormalities in the mitochondrial metabolism of carbon substrates for the generation of reducing equivalents (eg, the reduced forms of the electron transporters, Nicotinamide Adenine Dinucleotide and Flavin Adenine Dinucleotide: $\mathrm{NADH}$ and $\mathrm{FADH} 2$ ). Fatty acid oxidation provides significant energy for oxidative phosphorylation. ${ }^{46}$ Impaired oxidation of glucose and lactate may contribute to cardiac contractile dysfunction. ${ }^{47}$ Furthermore, electron microscopy has found mitochondria to be disrupted and damaged in failing hearts. ${ }^{48}$ Evidence supporting a causal link between impaired mitochondrial metabolic function and cardiac failure has been found in several cases of inherited cardiomyopathy. ${ }^{49}$ Cardiac mitochondria exist as two different populations, interfibrillar and subsarcolemmal, and these have been shown to respond differently to insults, for example, ageing, ischaemia and inherited cardiomyopathy. ${ }^{50-52}$

\section{DRUG TOXICITY AND MITOCHONDRIA}

Mitochondrial dysfunction has been increasingly implicated in drug-induced toxicity. Medications can damage mitochondria, both, directly and indirectly. For example, medications can directly inhibit mitochondrial DNA transcription of electron transport chain complexes, or inhibit enzymes required for glycolysis, or indirectly damage mitochondria by formation of free radicals, as with the anthracylines. Damage to mitochondria was first demonstrated in vitro with barbiturates inhibiting mitochondrial respiration. ${ }^{53}$ Damage to mitochondria is primarily caused by reactive oxygen species created by mitochondria themselves. ${ }^{54}$ Inflammatory mediators such as tumour necrosis factor $\alpha$ (TNF- $\alpha$ ) have been associated in vitro with mitochondrial dysfunction and increased radical oxygen species generation. ${ }^{55}$

\section{MITOCHONDRIA-DIRECTED THERAPY IN HEART FAILURE PATIENTS}

Approximately two-thirds of the energy required for oxidative phosphorylation is derived from the oxidation of fatty acids.

\section{Main messages}

- Not all forms of heart failure are relentlessly progressive

- Heart failure caused by toxins and tachycardia can show reversibility

- The underlying mechanisms involve abnormalities in mitochondrial respiration and calcium processing

- These mechanisms may provide useful targets for the development of novel drug therapies for heart failure.

\section{Current research questions}

- Can partial fatty acid oxidation inhibitors, like trimetazidine and ranolazine, be trialled for use in heart failure?

- How important is the genetic predisposition to alcoholinduced cardiac toxicity, and is there a genetic crossover with 'idiopathic'-dilated cardiomyopathy?

- Can key anthracycline modifications be developed and utilised clinically to reduce cardiac toxicity? 


\section{Key references}

- Robinson T, Smith A, Channer KS. Reversible heart failure: the role of inflammatory activation. Postgrad Med $J$ 2011;87:110-15.

- Lange RA, Hillis LD. Cardiovascular complications of cocaine use. N Engl J Med 2001;345:351-8.

- Moe GW, Naik G, Konig A, et al. Early and persistent activation of myocardial apoptosis, bax and caspases: Insights into mechanisms of progression of heart failure. Pathophysiology 2002;8:183-92.

- Schimmel KJ, Richel DJ, Van Den Brink RB, et al. Cardiotoxicity of cytotoxic drugs. Cancer Treatment Reviews 2004;30:181-91.

- Bersin RM, Wolfe C, Kwasman M, et al. Improved haemodynamic - function and mechanical efficiency in congestive heart failure with sodium dichloroacetate. J Am Coll Cardiol 1994;23:1617-24.

Patients with heart failure may have impaired metabolism of lactate and glucose, and enhanced fatty acid oxidation that contributes to cardiac dysfunction. ${ }^{46} 47$ Drugs aimed at increasing the myocardial oxidation of derivatives of glucose and lactate could be beneficial in heart failure patients. ${ }^{56}$ Pharmacological activation of pyruvate dehydrogenase has been demonstrated to increase left ventricular function without an increase in myocardial oxygen consumption. ${ }^{57}$ Partial fatty acid oxidation inhibitors increase pyruvate oxidation which removes product inhibition of pyruvate dehydrogenase. The partial fatty acid oxidation inhibitors, trimetazidine and ranolazine, have been used successfully for the treatment of angina. ${ }^{46}$ Their use in heart failure remains to be fully tested.

Existing heart failure therapies exert some of their therapeutic effects by their actions on mitochondria. For example, long-term treatment with the $\beta$-receptor antagonist, Metoprolol, has been demonstrated to reduce fatty acid oxidation and increase carbohydrate oxidation with an associated improvement in LVEF. $^{58}$ Studies in rats have demonstrated that ACE inhibitors, slow down heart failure progression following myocardial infarction in association with a fall in the rate of cardiac mitochondrial respiration..$^{59}$

\section{CONCLUSION}

Mitochondrial dysfunction appears to play a pivotal role in patients with toxin- and tachycardia-induced heart failure. In these circumstances, heart failure may demonstrate marked reversibility. Existing heart failure therapies already exert beneficial effects on mitochondrial function. Prompt identification of such cases, cessation of the causative toxic agent or restoration of normal heart rate and standard heart failure therapy remain the current treatment modalities. Trials of new agents specifically aimed at improving mitochondrial function in heart failure are ongoing.

\section{MULTIPLE CHOICE QUESTIONS (TRUE (T)/FALSE (F); ANSWERS AFTER THE REFERENCES)}

\section{Regarding alcohol and the heart}

A. Myocytes contain high levels of the enzyme alcohol dehydrogenase.

B. Any level of alcohol consumption is detrimental to cardiovascular morbidity and mortality.
C. Ethanol and acetaldehyde are directly toxic to actin and myosin protein filaments.

D. Toxicity to alcohol in the heart is influenced by predisposing genetic factors.

\section{Regarding sympathomimetic drugs and the heart}

A. Sympathomimetic drugs affect cardiac function acutely but not chronically.

B. Cocaine blocks the presynaptic uptake of catecholamines and dopamine.

C. Cocaine has more centrally acting effects than amphetamines.

D. Sympathomimetic drugs may induce coronary spasm which may result in myocardial infarction.

\section{Regarding tachycardia-mediated cardiomyopathy}

A. Tachycardia is a common cause of cardiomyopathy.

B. Is mediated by mitochondrial oxidative damage.

C. Treatment of tachycardic arrhythmia in cardiomyopathy frequently reverses the cardiomyopathy.

D. The combination of cardiomyopathy and arrhythmia should alert the physician to consider anticoagulation, if appropriate.

\section{Regarding chemotherapy and the heart}

A. Anthracyclines inhibit DNA synthesis.

B. Anthracycline use is associated with the generation of oxygen free radicals.

C. Cardiac myocytes contain relatively high amounts of antioxidant enzymes.

D. The ErbB2 protein appears to have a protective role in patients treated with trastuzumab.

\section{Regarding myocardial toxins and cardiomyopathy}

A. Cardiac myocytes contain relatively low numbers of mitochondria.

B. In the failing heart, mitochondrial function is activated and made more efficient.

C. Mitochondrial damage is primarily caused by reactive oxygen species created by mitochondria themselves.

D. Drugs aimed at improving the oxidation of derivatives of glucose and lactate may prove to be beneficial in heart failure patients in the future.

Acknowledgements We thank Dr Laurence 0'Toole for helpful comments and advice in preparing this manuscript.

Contributors Professor KSC conceived the original idea for this two-part review series. All three authors directly contributed to the text in this manuscript. Dr PM and Dr TR composed the article.

Competing interests None.

Provenance and peer review Not commissioned; externally peer reviewed.

\section{REFERENCES}

1. Levy D, Kenchaiah S, Larson MG, et al. Long-term trends in the incidence of and survival with heart failure. N Engl J Med 2002;347:1397-402.

2. Robinson T, Smith A, Channer KS. Reversible heart failure: the role of inflammatory activation. Postgrad Med J 2011;87:110-15.

3. Kokolis S, Marmur JD, Clark LT, et al. Effects of alcoholism on coronary artery disease and left ventricular dysfunction in male veterans. J Invasive Cardiol 2006;18:304-7.

4. Dancy M, Bland JM, Leech G, et al. Preclinical left ventricular abnormalities in alcoholics are independent of nutritional status, cirrhosis, and cigarette smoking. Lancet 1985;1:1122-5.

5. Bing RJ. Cardiac metabolism: its contributions to alcoholic heart disease and myocardial failure. Circulation 1978;58:965-70.

6. Arriola L, Martinez-Camblor P, Larrañaga N, et al. Alcohol intake and the risk of coronary heart disease in the Spanish EPIC cohort study. Heart 2010;96:124-30. 
7. Fernández-Solà J, Nicolás JM, Oriola J, et al. Angiotensin-converting enzyme gene polymorphism is associated with vulnerability to alcoholic cardiomyopathy. Ann Intern Med 2002;137:321-6

8. Institute for the Study of Drug Dependence. UK National Report for the European Monitoring Centre for Drugs and Drug addiction, London: Institute for the Study of Drug Dependence, 1997.

9. Afonso L, Mohammad T, Thatai D. Crack whips the heart: a review of the cardiovascular toxicity of cocaine. Am J Cardiol 2007;100:1040-3.

10. Mouhaffet A, Madu E, Satmary W, et al. Cardiovascular complications of cocaine. Chest 1995;107:1426-34.

11. Bertolet BD, Freund G, Martin CA, et al. Unrecognized left ventricular dysfunction in an apparently healthy cocaine abuse population. Clin Cardiol 1990;13:323-8.

12. Lange RA, Hillis LD. Cardiovascular complications of cocaine use. $N$ Engl J Med 2001;345:351-8.

13. Chiueh C, Kopin J. Centrally mediated release of cocaine by endogenous epinephrine and norepinephrine from the sympathoadrenal medullary system of unanesthetised rats. J Pharmacol Exp Ther 1978;205:148-54.

14. Burke WM, Ravi NV. Urinary excretion of cocaine. Ann Intern Med 1990;112:548-9.

15. Tonga G, Tempesta E. Platelet responsiveness and biosynthesis of thrombaxane and prostacyclin in response to in vitro cocaine treatment. Homeostasis 1985:15:100-7.

16. Tracy CM. Tachycardia-mediated cardiomyopathy. Up-To-Date 2002;10.3. http:// www.uptodate.com (accessed 17 Nov 2002).

17. Kasper EK, Agema WR, Hutchins GM, et al. The causes of dilated cardiomyopathy: a clinicopathologic review of 673 consecutive patients. J Am Coll Cardiol 1994;23:586-90.

18. Moe GW, Naik G, Konig A, et al. Early and persistent activation of myocardial apoptosis, bax and caspases: insights into mechanisms of progression of heart failure. Pathophysiology 2002;8:183-92.

19. Hanna N, Cardin S, Leung TK, et al. Differences in atrial versus ventricular remodeling in dogs with ventricular tachy pacing induced congestive heart failure. Cardiovasc Res 2004;63:236-44.

20. Meinardi MT, Gietema JA, van Veldhuisen DJ, et al. Long-term chemotherapy related cardiovascular morbidity. Cancer Treat Rev 2000;26:429-47

21. Arola $\mathbf{0 J}$, Saraste A, Pulkki K, et al. Acute doxorubicin cardiotoxicity involves cardiomyocite apoptosis. Cancer Res 2000:60:1789-92.

22. Hatch GM, McClarty G. Regulation of cardiolipin biosynthesis in H9c2 cardiac myoblasts by cytidine 50-triphosphate. J Biol Chem 1996:271:25810-16.

23. Von Hoff DD, Layard MW, Basa P, et al. Risk factors for doxorubicin-induced congestive heart failure. Ann Intern Med 1979;91:710-17.

24. Ewer MS, Lippman SM. Type II chemotherapy-related cardiac dysfunction: time to recognize a new entity. J Clin Oncol 2005;23:2900-2.

25. Rossi F, Fillippelli W, Russo S, et al. Cardiotoxicity of doxorubicin: effects of drugs inhibiting the release of vasoactive substances. Pharm Tox 1994; 75:99-107

26. Vasquez-Vivar J, Martasek $\mathrm{P}$, Hogg $\mathrm{N}$, et al. Endothelial nitric oxide synthase-dependent superoxide generation from adriamycin. Biochem 1997;36:11293-7

27. Rajagopalan S, Politi PM, Sinha BK, et al. Adriamycin induced free radical formation in the perfused rat heart: implications for cardiotoxicity. Cancer Res 1988;48:4766-9.

28. Doroshow JH, Locker GY, Myers CE. Enzymatic defenses of the mouse heart against reactive oxygen metabolites. J Clin Invest 1980;65:128-35.

29. Zhou S, Starkov A, Frober MK, et al. Cumulative and irreversible cardiac mitochondrial dysfunction induced by doxorubicin. Cancer Res 2001;61:771-7.

30. Schimmel KJ, Richel DJ, Van Den Brink RB, et al. Cardiotoxicity of cytotoxic drugs Cancer Treat Rev 2004:30:181-91.

31. Ryberg M, Nielsen D, Cortese G, et al. New insight into epirubicin cardiac toxicity: competing risks analysis of 1097 breast cancer patients. J Natl Cancer Inst 2008; 100:1058-67.

32. Ryberg M, Nielsen D, Skovsgaard T, et al. Epirubicin cardiotoxicity: an analysis of 469 patients with metastatic breast cancer. J Clin Oncol 1998;16:3502-8.

33. Fumoleau P, Roché H, Kerbrat $\mathrm{P}$, et al; French Adjuvant Study Group. Long-term cardiac toxicity after adjuvant epirubicin-based chemotherapy in early breast cancer: French Adjuvant Study Group results. Ann Oncol 2006;17:85-92.

34. Dorr R. Chemoprotectants for cancer chemotherapy. Semin Oncol 1991;18:48-58.

35. Myers CE, Bonow R, Palmeri S, et al. A randomised controlled trial assessing the prevention of doxorubicin cardiomyopathy by N-acetylcysteine. Semin Oncol 1983;10:53-5.

36. Swain WM, Whaley FS, Gerber MC, et al. Cardioprotection with dexrazoxane for doxorubicin-containing therapy in advanced breast cancer. J Clin Oncol 1997; 15:1318-32.
37. Speyer JL, Green MD, Kramer E, et al. Protective effect of the bispiperazinedione ICRF-187 against doxorubicin-induced cardiac toxicity in women with advanced breast cancer. N Engl J Med 1988;319:745-52.

38. Safra T, Muggia F, Jeffers $S$, et al. Pegylated liposomal doxorubicin (doxil): reduced clinical cardiotoxicity in patients reaching or exceeding cumulative doses of $500 \mathrm{mg} /$ m2. Ann Oncol 2000:11:1029-33.

39. Baselga J, Carbonell X, Castaneda-Soto NJ, et al. Phase II study of efficacy, safety, and pharmacokinetics of trastuzumab monotherapy administered $n$ a 3- weekly schedule. J Clin Oncol 2005:23:2162-71.

40. Vogel CL, Cobleigh MA, Tripathy D, et al. Efficacy and safety of trastuzumab as a single agent in first-line treatment of HER2-overexpressing metastatic breast cancer. J Clin Oncol 2002:20:719-26.

41. Chien KR. Focus on research: herceptin and the heart-a molecular modifier of cardiac failure. N Engl J Med 2006;354:789-90.

42. Crone SA, Zhao $Y Y$, Fan $L$, et al. ErbB2 is essential in the prevention of dilated cardiomyopathy. Nat Med 2002;8:459-65.

43. Chen $\mathbf{T}, \mathrm{Xu}$ T, Li Y, et al. Risk of cardiac dysfunction with trastuzumab in breast cancer patients: a meta-analysis. Cancer Treat Rev 2011;37:312-20.

44. Jones AL, Barlow M, Barrett-Lee PJ, et al. Management of cardiac health in trastuzumab-treated patients with breast cancer: updated United Kingdom Nationa Cancer Research Institute recommendations for monitoring. $\mathrm{Br} \mathrm{J}$ Cance 2009;100:684-92.

45. Bashore TM, Magorien DJ, Letterio J, et al. Histologic and biochemical correlates of left ventricular chamber in man. J Am Col Cardiol 1987;9:734-42.

46. Stanley WC, Lopaschuk GD, Hall JH, et al. Regulation of myocardial carbohydrate metabolism under normal and ischaemic conditions: potential for pharmacological interventions. Cardiovasc Res 1997;33:243-57.

47. Paolisso G, Gambardella A, Galzerano D, et al. Total body and myocardial substrate oxidation in congestive heart failure. Metabolism 1994;43:174-8.

48. Sabbah HN, Sharov VG, Riddle JM, et al. Mitochondrial abnormalities in myocardium of dogs with chronic heart failure. J Mol Cell Cardiol 1992:24:1333-47.

49. Kelly DP, Strauss AW. Inherited cardiomyopathies. N Engl J Med 1994;330:913-19.

50. Fannin SW, Lesnefsky EJ, Slabe TJ, et al. Aging selectively decreases oxidative capacity in rat heart interfibrillar mitochondria. Arch Biochem Biophys 2000;372:1.

51. Lesnefsky EJ, Tandler B, Ye J, et al. Myocardial ischemia decreases oxidative phosphorylation through cytochrome oxidase in subsarcolemmal mitochondria. Am J Physiol 1997;273:1544-54.

52. Hoppel CL, Tandler B, Parland W, et al. Hamster cardiomyopathy: a defect in oxidative phosphorylation in the cardiac interfibrillar mitochondria. J Biol Chem 1982;257:1540-8.

53. Chan K, Truong D, Shangari N, et al. Drug induced mitochondrial toxicity. Expert Opin Drug Metab Toxicol 2005;1:655-69.

54. Wei YH, Lu CY, Lee HC, et al. Oxidative damage and mutation to mitochondrial DNA and age dependent decline of mitochondrial respiratory function. Ann N Y Acad Sci 1998;854:155-70.

55. Moe GW, Marin-Garcia J, Konig A, et al. In vivo TNF- $\{$ alpha $\}$ inhibition ameliorates cardiac mitochondrial dysfunction, oxidative stress, and apoptosis in experimental heart failure. Am J Physiol Heart Circ Physiol 2004;287:H1813-20.

56. From AH. Should manipulation of myocardial substrate utilization patterns be a component of the congestive heart failure therapeutic paradigm? J Card Fail 1998:4:127-9.

57. Bersin RM, Wolfe C, Kwasman M, et al. Improved hemodynamic - function and mechanical efficiency in congestive heart failure with sodium dichloroacetate. J Am Coll Cardiol 1994;23:1617-24.

58. Eichhorn EJ, Heesch CM, Barnett JH, et al. Effect of metoprolol on myocardial function and energetics in patients with non-ischemic dilated cardiomyopathy: a randomized, double-blind, placebo controlled study. J Am Coll Cardiol 1994:24:1310-20.

59. Sanbe A, Tanonake K, Kobayasi R, et al. Effect of long-term therapy with ACE inhibitors on myocardial energy metabolism in rats with heart failure following myocardial infarction. J Mol Cell Cardiol 1995;27:2209-22.

\section{ANSWERS}

1. $A(F) ; B(F) ; C(F) ; D(T)$

2. $A(F) ; B(T) ; C(F) ; D(T)$

3. $A(F) ; B(T) ; C(F) ; D(T)$

4. $A(T) ; B(T) ; C(F) ; D(T)$

5. $A(F) ; B(F) ; C(T) ; D(T)$ 